\title{
Tests of fit for the asymmetric Laplace distribution
}

\author{
Colin Chen*
}

Tests based on the empirical distribution function (EDF) are given for the goodness-of-fit of the three-parameter asymmetric Laplace distribution. Asymptotic distributions of the test statistics are derived and their critical values are computed. For finite samples, simulated critical values of these tests are approximated by simple polynomial functions of the sample size and the shape parameter. Good matches between the asymptotic critical values and the extrapolated critical values from finite samples validate the procedure with finite samples. Power studies are reported to compare among these tests. The Anderson-Darling statistic $A^{2}$ gives the overall most powerful EDF tests followed by the Cramér-Von Mises statistic $W^{2}$.

AMS 2000 SUBJECT CLASSIFICATIONS: 62F03.

KEYWORDS AND PHRASES: Asymmetric Laplace distribution, Empirical distribution function, Goodness of fit, Maximum likelihood estimation.

\section{INTRODUCTION}

The asymmetric Laplace distribution becomes more and more popular in handling financial data, which present long tails and asymmetry. Hartley and Revankar (1974, [8]) reported that the logarithm of underreported incomes, property values, firm or city values, etc. follow asymmetric three-parameter Laplace distributions. Kozubowski and Podgórski (1999, [13]) modeled interest rates using an asymmetric Laplace distribution. Kozubowski and Podgórski (2001, [14]) presented an application of the asymmetric Laplace distribution in modeling foreign currency exchange rates. The Laplace motion, which has symmetric and asymmetric Laplace increments, was used to model stock market returns in Longstaff $(1994$, [15]) and Madan et al. $(1998,[16])$.

Goodness-of-fit tests based on EDF for the two-parameter symmetric Laplace distribution have been studied by Puig and Stephens (2000, [17]) and Chen (2002, [3]). While the first paper focuses more on the asymptotic distributions of the test statistics, the second paper focuses on distributions of the test statistics with finite samples. It was found that the asymptotic distributions of the test statistics depend

* Research was initiated when the author was with SAS Institute Inc. Cary, NC, USA. on the efficient estimators used in the tests and can be approximated by a sum of weighted independent chi-square distributions. For the finite sample case, the theoretical distributions of these test statistics do not have closed forms and Monte Carlo simulations were used to simulate the critical values of these distributions. These critical values only depend on the sample size. Polynomial functions of the sample size $n$ were developed to summarize these critical values for practical uses.

For the three-parameter asymmetric Laplace distribution, besides the location and scale parameters, there is a shape parameter. We show that both the asymptotic distributions and the finite-sample distributions of the EDF statistics depend on this shape parameter. The asymptotic distribution can be approximated by a sum of weighted independent chi-square distributions, where the weights depend on the shape parameter. The corresponding distributions with finite samples can be simulated using the Monte Carlo method. Polynomial functions based on the sample size and the shape parameter can be used to approximate the critical values of these distributions with high accuracy.

A goodness-of-fit test of the asymmetric Laplace distribution is a test of $H_{0}$ : a given random sample of $n$ values of $X$ comes from the distribution with a probability density function (p.d.f.)

(1) $f(x ; \theta, \sigma, \kappa)=\frac{\sqrt{2}}{\sigma} \frac{\kappa}{1+\kappa^{2}} \begin{cases}\exp \left(-\frac{\sqrt{2} \kappa}{\sigma}|x-\theta|\right) & x \geq \theta \\ \exp \left(-\frac{\sqrt{2}}{\kappa \sigma}|x-\theta|\right) & x<\theta,\end{cases}$

where $\theta$ is the location parameter, $\sigma$ is the scale parameter, and $\kappa$ acts as the shape parameter. The corresponding cumulative distribution function (c.d.f.) is

$$
F(x ; \theta, \sigma, \kappa)= \begin{cases}1-\frac{1}{1+\kappa^{2}} \exp \left(-\frac{\sqrt{2} \kappa}{\sigma}|x-\theta|\right) & x \geq \theta \\ \frac{\kappa^{2}}{1+\kappa^{2}} \exp \left(-\frac{\sqrt{2}}{\kappa \sigma}|x-\theta|\right) & x<\theta .\end{cases}
$$

This distribution is referred to as $A L(\theta, \sigma, \kappa)$ throughout the paper. We assume the sample has been sorted in increasing order, so $x_{1} \leq x_{2} \leq \cdots \leq x_{n}$. The alternate hypothesis $H_{1}$ is: the sample does not come from $A L(\theta, \sigma, \kappa)$.

In this paper, we examine the tests based on EDF statistics. According to the three parameters, Kotz et al. (2001, [12]) defined seven cases for estimation. We focus on the two most popular cases in applications of asymmetric Laplace distributions - the case with all three parameters 
unknown and the case with both scale and shape unknown while the location is known.

Case A. $\theta, \sigma$, and $\kappa$ are unknown,

Case B. $\sigma$ and $\kappa$ are unknown, $\theta$ is known.

EDF statistics depend on the method which is used to estimate the unknown parameters. Only the maximum likelihood estimators (MLEs) are used in this study. They are given in Section 2. In Section 3, the EDF statistics and their critical values approximated by simple polynomial functions based on simulated results from finite samples are given. Asymptotic critical values are derived in Section 4 and used to check the accuracy of the polynomial approximation in Section 3. In Section 5, three applications of the developed EDF tests are demonstrated. In Section 6, a power study is presented. These EDF tests are compared based on their power. Finally, some guidelines for using these techniques in practice are given in Section 7 .

\section{MAXIMUM LIKELIHOOD ESTIMATION}

The MLE of the three unknown parameters $\theta, \mu$, and $\sigma^{2}$ maximizes the likelihood function of a random sample consisting of observed ordered values $\boldsymbol{x}=\left(x_{1}, \ldots, x_{n}\right)$ from $n$ mutually independent random variables $\boldsymbol{X}=\left(X_{1}, \ldots, X_{n}\right)$, each with probability density function (1). The scaled loglikelihood function (divided by $n$ ) is

$$
\begin{aligned}
\frac{1}{n} \ln L(\boldsymbol{x} ; \theta, \sigma, \kappa)= & -\log \sigma+\log \left(\frac{\kappa}{1+\kappa^{2}}\right) \\
& -\frac{\sqrt{2}}{\sigma}\left(\kappa \alpha(\theta)+\frac{1}{\kappa} \beta(\theta)\right),
\end{aligned}
$$

where

$$
\begin{aligned}
& \alpha(\theta)=\frac{1}{n} \sum_{i=1}^{n}\left(x_{i}-\theta\right)^{+}, \\
& \beta(\theta)=\frac{1}{n} \sum_{i=1}^{n}\left(x_{i}-\theta\right)^{-}
\end{aligned}
$$

$(x)^{+}=x$ if $x \geq 0$ and 0 otherwise, and $(x)^{-}=-x$ if $x \leq 0$ and 0 otherwise.

For the three-parameter asymmetric Laplace distribution, with a different parametrization, Hartley and Revankar (1974, [8]) first derived the MLE and proposed an estimation procedure. Later, Hinkley and Revankar (1977, [9]) improved the estimation procedure and provided a complete proof of consistency, efficiency, and asymptotic normality of the MLE.

Define the function $h$ as

$$
h(\theta)=2 \log \left(\alpha(\theta)^{\frac{1}{2}}+\beta(\theta)^{\frac{1}{2}}\right)+\alpha(\theta)^{\frac{1}{2}} \beta(\theta)^{\frac{1}{2}} .
$$

Under the parametrization in (1), Kotz et al. (2001, [12]) (Section 3.5.1.7) describes the estimation procedure as follows:

Step 1. Evaluate the $n$ values $h\left(x_{i}\right), i=1, \ldots, n$ and choose a positive integer $r \leq n$ such that

$$
h\left(x_{r}\right) \leq h\left(x_{i}\right), i=1, \ldots, n .
$$

Step 2. If $r=1$ or $r=n$ then the MLE does not exist.

Step 3. If $1<r<n$, then the MLEs of $\theta, \sigma$ and $\kappa$ are

$$
\begin{aligned}
\hat{\theta} & =x_{r}, \\
\hat{\sigma} & =\sqrt{2} \alpha(\hat{\theta})^{\frac{1}{4}} \beta(\hat{\theta})^{\frac{1}{4}}\left(\alpha(\hat{\theta})^{\frac{1}{2}}+\beta(\hat{\theta})^{\frac{1}{2}}\right), \\
\hat{\kappa} & =\alpha(\hat{\theta})^{-\frac{1}{4}} \beta(\hat{\theta})^{\frac{1}{4}},
\end{aligned}
$$

where $\alpha$ and $\beta$ are functions as defined in (4).

When $\theta$ is known (Case B), the MLEs of $\sigma$ and $\kappa$ are the same as in (6) if $x_{1}<\theta<x_{n}$ and do not exist otherwise. The cases of $\theta \leq x_{1}$ or $\theta \geq x_{n}$ indicate that all sample points fall on one side of $\theta$, which lead to inadmissible MLEs of $\sigma=0, \kappa=0, \infty$ corresponding to exponential distributions. The cases $r=1$ or $r=n$ for the three-parameter MLE are similar.

\section{EDF STATISTICS AND TESTS}

EDF statistics are based on the discrepancy between distribution (2), with estimates used for $\theta, \sigma$, and $\kappa$, and the empirical distribution function of the sample of $x$ values. They are usually divided into two classes: the supremum and the quadratic. For the supremum class, this paper concentrates on the well-known Kolmogorov-Smirnov statistic $D$. For the quadratic class this paper concentrates on the Anderson-Darling statistic $A^{2}$ and the Cramér-Von Mises statistic $W^{2}$. Let $\hat{F}$ denote the distribution function in (2) with estimated $\theta, \sigma$, and $\kappa$. Let $F_{n}$ denote the empirical distribution function of the sample of $x$ values. The above mentioned statistics have the following formulae:

$$
\begin{aligned}
D & =\sup _{x}\left|F_{n}(x)-\hat{F}(x)\right| . \\
W^{2} & =n \int_{-\infty}^{\infty}\left\{F_{n}(x)-\hat{F}(x)\right\}^{2} \mathrm{~d} \hat{F}(x) . \\
A^{2} & =n \int_{-\infty}^{\infty} \frac{\left\{F_{n}(x)-\hat{F}(x)\right\}^{2}}{\hat{F}(x)\{1-\hat{F}(x)\}} \mathrm{d} \hat{F}(x) .
\end{aligned}
$$

Let $z_{i}=\hat{F}\left(x_{i}\right)\left(z_{i}, i=1, \ldots, n\right.$ are ordered since $\hat{F}$ is monotone and $x_{i}$ are ordered), then

$$
\begin{aligned}
D^{+}= & \max _{i}\left\{\frac{i}{n}-z_{i}\right\} ; \\
D^{-}= & \max _{i}\left\{z_{i}-\frac{(i-1)}{n}\right\} ; \\
D= & \max \left(D^{+}, D^{-}\right) ; \\
W^{2}= & \sum_{i}\left\{z_{i}-\frac{(2 i-1)}{2 n}\right\}^{2}+\frac{1}{12 n} ; \\
A^{2}= & -n-\frac{1}{n} \sum_{i}\left[(2 i-1) \ln \left(z_{i}\right)\right. \\
& \left.+(2 n+1-2 i) \ln \left\{1-z_{i}\right\}\right] .
\end{aligned}
$$


The algorithm in the previous section computes the MLEs, $\hat{\theta}, \hat{\sigma}$, and $\hat{\kappa}$, then $z_{i}=\hat{F}\left(x_{i}\right)=F\left(x_{i} ; \hat{\theta}, \hat{\sigma}, \hat{\kappa}\right)$, and these statistics can be computed.

For the finite sample, the distributions of EDF statistics depend on the sample size $n$ and the true value of the shape parameter $\kappa$, but not the location and scale parameters because of the well-known location-scale invariance of these statistics (Stephens, 1986, [18]). Simulations can be done to obtain simulated critical values for these statistics. For a simple example, we simulated critical values for $\kappa=\{0.5,0.6,0.7,0.8,0.9,1.0\}$ and $n=\{50,100,150,200,250,300,400,500,750,1,000\}$ with 100,000 replications. Critical values corresponding to the following 11 test levels $\{0.5,0.25,0.20,0.15,0.10,0.05,0.025$, $0.01,0.005,0.0025,0.001\}$ were computed.

Plots of these critical values against sample size $n$ and shape parameter $\kappa$ show very clear patterns. Polynomial functions of sample size $n$ and shape parameter $\kappa$ can be used to fit the logarithm of critical values. The terms and coefficients of the fitted polynomial functions are given in Table 1. The $R^{2}$ s for the three fits are $0.999347(\sqrt{n} D)$, $0.999104\left(W^{2}\right)$, and $0.999661\left(A^{2}\right)$. To compute a critical value, one can use the fomula:

$$
C V_{\alpha}=\exp \left(b_{0}(\alpha)+b_{1}(\alpha) \kappa+b_{2}(\alpha) / \kappa+b_{3}(\alpha) / n\right),
$$

where $b_{0}(\alpha), b_{1}(\alpha), b_{2}(\alpha)$, and $b_{3}(\alpha)$ are the tabulated values at test level $\alpha$ in Table $1, n$ is the sample size, and $\kappa$ is the shape parameter which usually needs to be estimated.

Using these polynomial functions one can also get the limiting critical values by extrapolation $(n \rightarrow \infty)$. These limiting critical values will be checked by the asymptotic results later, and they are highly consistent with the asymptotic results. An advantage of these functions is the convenience with which they can be incorporated into software to compute $p$-values for goodness-of-fit tests based on EDF statistics.

\section{ASYMPTOTIC THEORY OF EDF TESTS}

In this section the asymptotic distributions of EDF statistics are derived. The derivation follows a well known procedure given by Durbin $(1973,[4])$. The regularity conditions stated in his paper are fully satisfied by the three-parameter asymmetric Laplace distribution. Under these conditions $y_{n}(z)=\sqrt{n}\left\{\hat{F}_{n}(z)-z\right\}$, where $\hat{F}_{n}(z)$ is the EDF of $z_{i}, i=$ $1, \ldots, n$, tends to a Gaussian process $y(z)$ as $n \rightarrow \infty$, and the EDF statistics are functionals of this process. The mean of $y(z)$ is 0 . To derive the distributions of these functionals, the covariance function $\rho(s, t)=E\{y(s) y(t)\}$ is needed. Durbin $(1973,[4])$ proved that if the estimators of $\theta, \sigma$, and $\kappa$ are efficient, then

$$
\rho(s, t)=\min \{s, t\}-s t-[g(s)]^{\prime} \Sigma[g(t)],
$$

where $g(s)=\left(g_{1}(s), g_{2}(s), g_{3}(s)\right)^{\prime}$ is the gradient of $F\left(x ; \eta_{1}, \eta_{2}, \eta_{3}\right)$ with respect to $\eta_{1}, \eta_{2}$ and $\eta_{3}$ evaluated at

\begin{tabular}{|c|c|c|c|c|}
\hline \multicolumn{5}{|l|}{$\overline{\sqrt{n} D}$} \\
\hline \multirow[b]{2}{*}{$\alpha$} & \multicolumn{4}{|c|}{ Terms in the Polynomial Functions } \\
\hline & 1 & $\kappa$ & $\frac{1}{\kappa}$ & $1 / n$ \\
\hline .50 & -0.821616 & 0.110733 & 0.150554 & -2.341142 \\
\hline .75 & -0.667445 & 0.086715 & 0.171068 & -2.126541 \\
\hline .80 & -0.616127 & 0.072783 & 0.171647 & -2.135732 \\
\hline .85 & -0.545402 & 0.048458 & 0.168832 & -2.052918 \\
\hline .90 & -0.457722 & 0.019696 & 165251 & -1.954576 \\
\hline .95 & -0.312306 & -0.032479 & 153531 & -1.866498 \\
\hline .975 & -0.186344 & -0.076483 & 0.141578 & -1.840812 \\
\hline .990 & -0.009488 & -0.149451 & 115461 & -1.639882 \\
\hline .995 & 0.079898 & -0.178182 & 106752 & -1.526147 \\
\hline .9975 & 0.164815 & -0.206768 & 097597 & -1.460131 \\
\hline .999 & 0.265690 & -0.238102 & 0.086205 & -1.468195 \\
\hline \multicolumn{5}{|l|}{$\overline{W^{2}}$} \\
\hline & \multicolumn{4}{|c|}{ Terms in the Polynomial Functions } \\
\hline$\alpha$ & 1 & $\kappa$ & $\frac{1}{\kappa}$ & $1 / n$ \\
\hline .50 & -3.922660 & 0.331541 & 0.392890 & -1.072349 \\
\hline .75 & -3.702958 & 491 & 495047 & 38308 \\
\hline .80 & -3.619466 & 0.315954 & 512420 & -1.171374 \\
\hline .85 & -3.510712 & 0.284082 & .529455 & -1.077013 \\
\hline .90 & -3.336930 & 0.218464 & 0.539065 & -1.043878 \\
\hline .95 & -3.038332 & 336 & 537379 & -0.809731 \\
\hline .975 & -2.750170 & -0.035115 & .524177 & -0.817428 \\
\hline .990 & -2.341382 & -0.232190 & 0.478302 & -0.498997 \\
\hline .995 & -2.090467 & -0.3 & 451768 & -0.144769 \\
\hline .9975 & -1.7 & -0.5 & 393600 & 0.574368 \\
\hline .999 & -1.515785 & -0.615405 & 0.365488 & 0.203477 \\
\hline \multicolumn{5}{|l|}{$A^{2}$} \\
\hline & \multicolumn{4}{|c|}{ Terms in the Polynomial Functions } \\
\hline$\alpha$ & 1 & $\kappa$ & $\frac{1}{\kappa}$ & $\overline{1 / n}$ \\
\hline .50 & -1.669934 & 0.200295 & 219514 & -0.938923 \\
\hline .75 & -1.537774 & 0.263341 & 0.310546 & -0.839992 \\
\hline .80 & -1.50 & 6918 & 335902 & -0.773883 \\
\hline .85 & -1.460271 & 0.287451 & 0.364397 & -0.862476 \\
\hline .90 & -1.406131 & 0.302058 & 0.401533 & -0.860105 \\
\hline .95 & -1.288568 & 0.293535 & 0.446627 & -0.933113 \\
\hline .975 & -1.138248 & 0.251836 & 0.469440 & -0.929486 \\
\hline .990 & -0.922275 & 0.176210 & 0.478656 & -0.630208 \\
\hline .995 & -0.756579 & 0.111753 & 0.476150 & -0.217956 \\
\hline .9975 & -0.587147 & 0.043333 & 0.464951 & 0.432553 \\
\hline .999 & -0.408987 & -0.023838 & 0.461166 & 0.892380 \\
\hline
\end{tabular}

Table 1. Coefficients $(n \geq 50,0.5 \leq \kappa \leq 1.0)$

$x=F^{-1}(s ; \theta, \sigma, \kappa), \eta_{1}=\theta, \eta_{2}=\sigma$, and $\eta_{3}=\kappa$. That is, denoting $\boldsymbol{\eta}=\left(\eta_{1}, \eta_{2}, \eta_{3}\right)=(\theta, \sigma, \kappa)$,

$$
g_{i}(s)=\left.\frac{\partial F(x ; \boldsymbol{\eta})}{\partial \eta_{i}}\right|_{x=F^{-1}(s ; \theta, \sigma, \kappa), \boldsymbol{\eta}=(\theta, \sigma, \kappa)}
$$

for $i=1,2,3$. The matrix $\Sigma$ is the inverse of the Fisher information matrix $I$ with entries

$$
I_{i j}=E\left[\frac{\partial\{\ln f(X ; \boldsymbol{\eta})\}}{\partial \eta_{i}} \cdot \frac{\partial\{\ln f(X ; \boldsymbol{\eta})\}}{\partial \eta_{j}}\right] .
$$

Tests of fit for the asymmetric Laplace distribution 407 
Let $\Phi_{\kappa}$ and $\varphi_{\kappa}$ be the c.d.f. and p.d.f. of the standard asymmetric Laplace variable $Z=\frac{X-\theta}{\sigma}$ respectively, i.e.,

$$
\Phi_{\kappa}(z)= \begin{cases}1-\frac{1}{1+\kappa^{2}} \exp (-\sqrt{2} \kappa z) & z \geq 0 \\ \frac{\kappa^{2}}{1+\kappa^{2}} \exp \left(\frac{\sqrt{2}}{\kappa} z\right) & z<0\end{cases}
$$

$$
\varphi_{\kappa}(z)=\frac{\sqrt{2} \kappa}{1+\kappa^{2}} \begin{cases}\exp (-\sqrt{2} \kappa z) & z \geq 0 \\ \exp \left(\frac{\sqrt{2}}{\kappa} z\right) & z<0\end{cases}
$$

so $s=F(x ; \theta, \sigma, \kappa)=\Phi_{\kappa}(z)$, and $z=\Phi_{\kappa}{ }^{-1}(s)$. As functions of $s$,

$$
\begin{aligned}
g_{i}(s) & =\frac{\partial F(x ; \boldsymbol{\eta})}{\partial \eta_{i}} \\
& =\frac{\partial \Phi_{\kappa}(z)}{\partial z} \frac{\partial z}{\partial \eta_{i}} \\
& =\varphi_{\kappa}\left(\Phi_{\kappa}^{-1}(s)\right) \frac{\partial z}{\partial \eta_{i}}, \quad i=1,2 . \\
g_{3}(s) & =\frac{\partial \Phi_{\kappa}(z)}{\partial \kappa} \\
& =\varphi_{\kappa}\left(\Phi_{\kappa}^{-1}(s)\right)\left(\frac{\sqrt{2}}{1+\kappa^{2}}+\frac{\left|\Phi_{\kappa}^{-1}(s)\right|}{\kappa}\right) .
\end{aligned}
$$

So, $g(s)=\varphi_{\kappa}\left(\Phi_{\kappa}^{-1}(s)\right) \sigma^{-1}\left(-1,-\Phi_{\kappa}^{-1}(s), \sigma\left(\frac{\sqrt{2}}{1+\kappa^{2}}+\right.\right.$ $\left.\left.\frac{\left|\Phi_{\kappa}^{-1}(s)\right|}{\kappa}\right)\right)^{\prime}$. The Fisher information matrix $I$ can be computed directly:

$$
I=\left[\begin{array}{ccc}
\frac{2}{\sigma^{2}} & -\frac{\sqrt{2}}{\sigma} \frac{2}{1+\kappa^{2}} & 0 \\
-\frac{\sqrt{2}}{\sigma} \frac{2}{1+\kappa^{2}} & \frac{1}{\kappa^{2}}+\frac{4}{\left(1+\kappa^{2}\right)^{2}} & -\frac{1}{\sigma \kappa} \frac{1-\kappa^{2}}{1+\kappa^{2}} \\
0 & -\frac{1}{\sigma \kappa} \frac{1-\kappa^{2}}{1+\kappa^{2}} & \frac{1}{\sigma^{2}}
\end{array}\right]
$$

and

$$
\begin{aligned}
& I^{-1}= \\
& {\left[\begin{array}{ccc}
\sigma^{2} & \frac{\sqrt{2}}{4} \sigma\left(1+\kappa^{2}\right) & \frac{\sqrt{2}}{4} \frac{\sigma^{2}}{\kappa}\left(1-\kappa^{2}\right) \\
\frac{\sqrt{2}}{4} \sigma\left(1+\kappa^{2}\right) & \frac{\left(1+\kappa^{2}\right)^{2}}{4} & \frac{\sigma}{4 \kappa}\left(1-\kappa^{2}\right)\left(1+\kappa^{2}\right) \\
\frac{\sqrt{2}}{4} \frac{\sigma^{2}}{\kappa}\left(1-\kappa^{2}\right) & \frac{\sigma}{4 \kappa}\left(1-\kappa^{2}\right)\left(1+\kappa^{2}\right) & \frac{\sigma^{2}}{4} \frac{\left(1+\kappa^{2}\right)^{2}}{\kappa^{2}}
\end{array}\right]}
\end{aligned}
$$

Substitute $g(s)$ and $\Sigma=I^{-1}$ into the covariance function $\rho(s, t)$, to get

$$
\begin{aligned}
& \rho(s, t)=\min \{s, t\}-s t \\
& -\left(\frac{1}{2}+B_{\kappa}(s, t)\right) \varphi_{\kappa}\left(\Phi_{\kappa}^{-1}(s)\right) \varphi_{\kappa}\left(\Phi_{\kappa}^{-1}(t)\right),
\end{aligned}
$$

where

$$
\begin{aligned}
& B_{\kappa}(s, t)= \\
& \begin{cases}\left(1+\kappa^{2}\right) \Phi_{\kappa}^{-1}(s) \Phi_{\kappa}^{-1}(t) & \frac{\kappa^{2}}{1+\kappa^{2}} \leq s \leq 1 \text { and } \frac{\kappa^{2}}{1+\kappa^{2}} \leq t \leq 1 \\
\left(1+\frac{1}{\kappa^{2}}\right) \Phi_{\kappa}^{-1}(s) \Phi_{\kappa}^{-1}(t) & 0 \leq s \leq \frac{\kappa^{2}}{1+\kappa^{2}} \text { and } 0 \leq t \leq \frac{\kappa^{2}}{1+\kappa^{2}} \\
0 & \text { otherwise. }\end{cases}
\end{aligned}
$$

It is immediately seen that $\rho(s, t)$ does not depend on $\theta$, $\sigma$, but on $\kappa$.
When $\theta$ is known, similarly we have the gradient of $F(x ; \sigma, \kappa), g_{\sigma, \kappa}(s)=\varphi_{\kappa}\left(\Phi_{\kappa}^{-1}(s)\right) \sigma^{-1}\left(-\Phi_{\kappa}^{-1}(s), \sigma\left(\frac{\sqrt{2}}{1+\kappa^{2}}+\right.\right.$ $\left.\left.\frac{\left|\Phi_{\kappa}^{-1}(s)\right|}{\kappa}\right)\right)^{\prime}$. The Fisher information matrix is

$$
I_{\sigma, \kappa}=\left[\begin{array}{cc}
\frac{1}{\sigma^{2}} & -\frac{1}{\sigma \kappa} \frac{1-\kappa^{2}}{1+\kappa^{2}} \\
-\frac{1}{\sigma \kappa} \frac{1-\kappa^{2}}{1+\kappa^{2}} & \frac{1}{\kappa^{2}}+\frac{4}{\left(1+\kappa^{2}\right)^{2}}
\end{array}\right]
$$

and

$$
I_{\sigma, \kappa}^{-1}=\frac{\sigma^{2}\left(1+\kappa^{2}\right)^{2}}{8}\left[\begin{array}{cc}
\frac{1}{\kappa^{2}}+\frac{4}{\left(1+\kappa^{2}\right)^{2}} & \frac{1}{\sigma \kappa} \frac{1-\kappa^{2}}{1+\kappa^{2}} \\
\frac{1}{\sigma \kappa} \frac{1-\kappa^{2}}{1+\kappa^{2}} & \frac{1}{\sigma^{2}}
\end{array}\right]
$$

Substitute $g_{\sigma, \kappa}(s)$ and $\Sigma=I_{\sigma, \kappa}^{-1}$ into the covariance function $\rho(s, t)$, to get

$$
\begin{aligned}
& \rho_{1}(s, t)=\min \{s, t\}-s t \\
& -\left(\frac{1}{4}+D_{\kappa}(s, t)\right) \varphi_{\kappa}\left(\Phi_{\kappa}^{-1}(s)\right) \varphi_{\kappa}\left(\Phi_{\kappa}^{-1}(t)\right),
\end{aligned}
$$

where $D_{\kappa}(s, t)$ can be computed from:

$$
\begin{aligned}
& \frac{\sqrt{2}}{4} \kappa\left(\Phi_{\kappa}^{-1}(s)+\Phi_{\kappa}^{-1}(t)\right) \\
& \quad+\left(1+\frac{\kappa^{2}}{2}\right) \Phi_{\kappa}^{-1}(s) \Phi_{\kappa}^{-1}(t), s, t \in\left(\frac{\kappa^{2}}{1+\kappa^{2}}, 1\right) ; \\
& \frac{\sqrt{2}}{4}\left(\kappa \Phi_{\kappa}^{-1}(s)+\frac{\Phi_{\kappa}^{-1}(t)}{\kappa}\right) \\
& \quad+\frac{1}{2} \Phi_{\kappa}^{-1}(s) \Phi_{\kappa}^{-1}(t), s \in\left(\frac{\kappa^{2}}{1+\kappa^{2}}, 1\right), t \in\left(0, \frac{\kappa^{2}}{1+\kappa^{2}}\right) ; \\
& \frac{\sqrt{2}}{4}\left(\frac{\Phi_{\kappa}^{-1}(s)}{\kappa}+\kappa \Phi_{\kappa}^{-1}(t)\right) \\
& \quad+\frac{1}{2} \Phi_{\kappa}^{-1}(s) \Phi_{\kappa}^{-1}(t), t \in\left(\frac{\kappa^{2}}{1+\kappa^{2}}, 1\right), s \in\left(0, \frac{\kappa^{2}}{1+\kappa^{2}}\right) ; \\
& -\frac{\sqrt{2}}{4 \kappa}\left(\Phi_{\kappa}^{-1}(s)+\Phi_{\kappa}^{-1}(t)\right) \\
& \quad+\left(1+\frac{1}{2 \kappa^{2}}\right) \Phi_{\kappa}^{-1}(s) \Phi_{\kappa}^{-1}(t), s, t \in\left(0, \frac{\kappa^{2}}{1+\kappa^{2}}\right) .
\end{aligned}
$$

The EDF statistics $W^{2}$ and $A^{2}$ tend to limiting statistics $W_{\infty}^{2}$ and $A_{\infty}^{2}$ as $n \rightarrow \infty$, respectively. $W_{\infty}^{2}$ and $A_{\infty}^{2}$ are functionals of the limiting process $y(z)$ :

$$
\begin{aligned}
W_{\infty}^{2} & =\int_{0}^{1} y^{2}(z) \mathrm{d} z \\
A_{\infty}^{2} & =\int_{0}^{1} \frac{y^{2}(z)}{z(1-z)} \mathrm{d} z .
\end{aligned}
$$

The distributions of the above statistics are sums of weighted independent $\chi_{1}^{2}$-variables, $\sum_{i=1}^{\infty} w_{i} \chi^{2}$. The weights $w_{i}$ (in descending order) are the eigenvalues of the system:

$$
w_{i} f_{i}(s)=\int_{0}^{1} \gamma(s, t) f_{i}(t) \mathrm{d} t
$$

where $f_{i}$ are the normalized eigenfunctions and $\gamma(s, t)=$ $\rho(s, t)$ for $W_{\infty}^{2}$ and $\gamma(s, t)=\rho(s, t) /\{s t(1-s)(1-t)\}^{\frac{1}{2}}$ for $A_{\infty}^{2}$. Equation (24) might be solved by numerical methods. Once the weights are known, the critical points of the distributions can be computed by the method of Imhof (1961, [10]). Table 2 and Table 3 show the asymptotic critical values of $W^{2}$ computed with $\kappa=0.1$ to 1.0 by 0.1 for 
Table 2. Asymptotic Critical Values of $W^{2}$ for Case $A$

\begin{tabular}{lrrrrr}
\hline \hline & \multicolumn{4}{c}{$\kappa$} & \\
\cline { 2 - 6 }$\alpha$ & 0.1 & 0.2 & 0.3 & 0.4 & 0.5 \\
\hline .50 & 0.073049 & 0.068696 & 0.063049 & 0.056893 & 0.051261 \\
.75 & 0.114652 & 0.107725 & 0.098581 & 0.088269 & 0.078297 \\
.80 & 0.128413 & 0.120635 & 0.110334 & 0.098646 & 0.087233 \\
.85 & 0.146521 & 0.137625 & 0.125801 & 0.112302 & 0.098992 \\
.90 & 0.172754 & 0.162238 & 0.148207 & 0.132086 & 0.116026 \\
.95 & 0.219391 & 0.205994 & 0.188041 & 0.167256 & 0.146310 \\
.975 & 0.267932 & 0.251538 & 0.229503 & 0.203864 & 0.177832 \\
.990 & 0.334314 & 0.313821 & 0.286203 & 0.253928 & 0.220943 \\
.995 & 0.385769 & 0.362098 & 0.330153 & 0.292733 & 0.254361 \\
.9975 & 0.438014 & 0.411117 & 0.374778 & 0.332135 & 0.288292 \\
.999 & 0.508002 & 0.476782 & 0.434558 & 0.384918 & 0.333747 \\
\hline & & & $\kappa$ & & \\
$\alpha$ & 0.6 & 0.7 & 0.8 & 0.9 & 1.0 \\
\hline .50 & 0.046872 & 0.043944 & 0.042238 & 0.041389 & 0.041154 \\
.75 & 0.069821 & 0.063585 & 0.059731 & 0.057790 & 0.057243 \\
.80 & 0.077371 & 0.069929 & 0.065223 & 0.062832 & 0.062155 \\
.85 & 0.087299 & 0.078229 & 0.072318 & 0.069270 & 0.068403 \\
.90 & 0.101678 & 0.090204 & 0.082415 & 0.078294 & 0.077109 \\
.95 & 0.127242 & 0.111451 & 0.100070 & 0.093725 & 0.091859 \\
.975 & 0.153857 & 0.133572 & 0.118285 & 0.109271 & 0.106538 \\
.990 & 0.190263 & 0.163853 & 0.143151 & 0.130073 & 0.125907 \\
.995 & 0.218487 & 0.187342 & 0.162449 & 0.146010 & 0.140550 \\
.9975 & 0.247146 & 0.211201 & 0.182071 & 0.162113 & 0.155191 \\
.999 & 0.285542 & 0.243173 & 0.208392 & 0.183633 & 0.174543 \\
\hline & & & & &
\end{tabular}

Case A and Case B respectively. When computing the quantiles of $\sum_{i=1}^{\infty} w_{i} \chi^{2}$ with the method of Imhof (1961, [10]), the sum of the first 200 terms was used as an approximation, i.e., $\sum_{i=1}^{200} w_{i} \chi^{2}$, to make sure the computed asymptotic critical values have sufficient precision.

The following facts show that the asymptotic distributions of $W^{2}(\kappa)$ and $W^{2}\left(\frac{1}{\kappa}\right)$ are the same. The same is true with $A^{2}(\kappa)$ and $A^{2}\left(\frac{1}{\kappa}\right)$. So Table 2 and Table 3 can also be used for $\kappa \in(1,10)$.

Lemma 1. For any $\kappa>0, s \in(0,1)$, and $t \in(0,1)$ the covariance functions $\rho(s, t)$ and $\rho_{1}(s, t)$ satisfy:

$$
\begin{aligned}
\rho(\kappa ; s, t) & =\rho\left(\frac{1}{\kappa} ; 1-s, 1-t\right) \\
\rho_{1}(\kappa ; s, t) & =\rho_{1}\left(\frac{1}{\kappa} ; 1-s, 1-t\right)
\end{aligned}
$$

Proof. For $s \in(0,1)$ and $t \in(0,1)$, let $\rho_{0}(s, t)=\min (s, t)-$ st. From (20) and (23),

$$
\begin{gathered}
\rho(\kappa ; s, t)=\rho_{0}(s, t)-\left(\frac{1}{2}+B_{\kappa}(s, t)\right) \varphi_{\kappa}\left(\Phi_{\kappa}^{-1}(s)\right) \varphi_{\kappa}\left(\Phi_{\kappa}^{-1}(t)\right), \\
\rho_{1}(\kappa ; s, t)=\rho_{0}(s, t)-\left(\frac{1}{4}+D_{\kappa}(s, t)\right) \varphi_{\kappa}\left(\Phi_{\kappa}^{-1}(s)\right) \varphi_{\kappa}\left(\Phi_{\kappa}^{-1}(t)\right) .
\end{gathered}
$$

\begin{tabular}{|c|c|c|c|c|c|}
\hline \multirow[b]{2}{*}{$\alpha$} & \multicolumn{5}{|c|}{$\kappa$} \\
\hline & 0.1 & 0.2 & 0.3 & 0.4 & 0.5 \\
\hline .50 & 0.073426 & 0.071422 & 0.068593 & 0.065496 & 0.062620 \\
\hline .75 & 0.115224 & 0.112039 & 0.107449 & 0.102271 & 0.097267 \\
\hline .80 & 0.129050 & 0.125475 & 0.120307 & 0.114446 & 0.108746 \\
\hline .85 & 0.147245 & 0.143157 & 0.137229 & 0.130476 & 0.123867 \\
\hline .90 & 0.173604 & 0.168775 & 0.161751 & 0.153713 & 0.145804 \\
\hline .95 & 0.220464 & 0.214321 & 0.205356 & 0.195053 & 0.184863 \\
\hline .975 & 0.269239 & 0.261728 & 0.250749 & 0.238104 & 0.225563 \\
\hline .990 & 0.335941 & 0.326562 & 0.312832 & 0.296989 & 0.281245 \\
\hline .995 & 0.387643 & 0.376815 & 0.360953 & 0.342632 & 0.324405 \\
\hline .9975 & 0.440140 & 0.427840 & 0.409813 & 0.388975 & 0.368225 \\
\hline \multirow[t]{2}{*}{.999} & 0.510465 & 0.496194 & 0.475265 & 0.451053 & 0.426919 \\
\hline & \multicolumn{5}{|c|}{$\kappa$} \\
\hline$\alpha$ & 0.6 & 0.7 & 0.8 & 0.9 & $\overline{1.0}$ \\
\hline .50 & 0.060275 & 0.058577 & 0.057495 & 0.056927 & 0.056761 \\
\hline .75 & 0.092994 & 0.089746 & 0.087587 & 0.086423 & 0.086078 \\
\hline .80 & 0.103840 & 0.100081 & 0.097563 & 0.096197 & 0.095790 \\
\hline .85 & 0.118139 & 0.113717 & 0.110734 & 0.109106 & 0.108621 \\
\hline .90 & 0.138904 & 0.133542 & 0.129902 & 0.127907 & 0.127310 \\
\hline .95 & 0.175924 & 0.168937 & 0.164173 & 0.161551 & 0.160766 \\
\hline .975 & 0.214531 & 0.205888 & 0.199982 & 0.196729 & 0.195754 \\
\hline .990 & 0.267366 & 0.256472 & 0.249017 & 0.244907 & 0.243674 \\
\hline .995 & 0.308319 & 0.295679 & 0.287023 & 0.282247 & 0.280815 \\
\hline .9975 & 0.349895 & 0.335480 & 0.325600 & 0.320147 & 0.318511 \\
\hline .999 & 0.405580 & 0.388782 & 0.377261 & 0.370898 & 0.368989 \\
\hline
\end{tabular}

Direct calculation shows that $B_{\kappa}(s, t)=B_{\frac{1}{\kappa}}(1-s, 1-t)$, $D_{\kappa}(s, t)=D_{\frac{1}{\kappa}}(1-s, 1-t)$, and $\varphi_{\kappa}\left(\Phi_{\kappa}^{-1}(s)\right)=\varphi_{\frac{1}{\kappa}}\left(\Phi_{\frac{1}{\kappa}}^{-1}(1-\right.$
Table 3. Asymptotic Critical Values of $W^{2}$ for Case $B$

$s)$ ). Since $\rho_{0}(s, t)=\rho_{0}(1-s, 1-t)$, we have $(25)$ and $(26)$ by plugging in these equations.

Lemma 2. For both Case $A$ and $B, \gamma(\kappa ; s, t)$ and $\gamma\left(\frac{1}{\kappa} ; s, t\right)$ have same eigenvalues as defined in (24). So, for any $\kappa>0$, The asymptotic distributions of $W^{2}(\kappa)$ and $W^{2}\left(\frac{1}{\kappa}\right)$ are the same. The same is true with $A^{2}(\kappa)$ and $A^{2}\left(\frac{1}{\kappa}\right)$.

Proof. For $W^{2}, \gamma(\kappa ; s, t)=\rho(\kappa ; s, t)$, so $\gamma(\kappa ; s, t)=\gamma\left(\frac{1}{\kappa} ; 1-\right.$ $s, 1-t$ ) from Lemma 1 . Let $u=1-s$ and $v=1-t$, from (24) we have

$$
\begin{aligned}
w_{i} f_{i}(1-u) & =\int_{0}^{1} \gamma(\kappa ; s, t) f_{i}(t) \mathrm{d} t \\
& =\int_{0}^{1} \gamma\left(\frac{1}{\kappa} ; 1-s, 1-t\right) f_{i}(t) \mathrm{d} t \\
& =\int_{0}^{1} \gamma\left(\frac{1}{\kappa} ; u, v\right) f_{i}(1-v) \mathrm{d} v
\end{aligned}
$$

Again, by the definition (24), $w_{i}$ is also an eigenvalue of $\gamma\left(\frac{1}{\kappa} ; u, v\right)$ with the eigenfunction $f_{i}(1-u)$. So, $\gamma(\kappa ; s, t)$ and $\gamma\left(\frac{1}{\kappa} ; s, t\right)$ have same eigenvalues. Since the asymptotic distribution of $W^{2}(\kappa)$ is the sum of weighted independent $\chi_{1}^{2}$-variables, $\sum_{i=1}^{\infty} w_{i} \chi^{2}$, thus the asymptotic distributions of $W^{2}(\kappa)$ and $W^{2}\left(\frac{1}{\kappa}\right)$ are the same. For $A^{2}, \gamma(\kappa ; s, t)=$ $\gamma\left(\frac{1}{\kappa} ; 1-s, 1-t\right)$, so the same is true with $A^{2}(\kappa)$ and $A^{2}\left(\frac{1}{\kappa}\right)$.

Tests of fit for the asymmetric Laplace distribution 409 
Table 4. Asymptotic and Simulated (by Extrapolation) Critical Values for $W^{2}$

\begin{tabular}{|c|c|c|c|}
\hline$\alpha$ & Asymptotic & Extrapolation & Relative Diff. $\times 100$ \\
\hline \multicolumn{4}{|l|}{$\bar{\kappa}=.5$} \\
\hline .50 & 0.05126 & 0.05119 & 0.1365 \\
\hline .75 & 0.07829 & 0.07837 & 0.1021 \\
\hline .80 & 0.08723 & 0.08735 & 0.1375 \\
\hline .85 & 0.09899 & 0.09918 & 0.1919 \\
\hline .90 & 0.11602 & 0.11641 & 0.3361 \\
\hline .95 & 0.14631 & 0.14691 & 0.4100 \\
\hline .975 & 0.17783 & 0.17903 & 0.6748 \\
\hline .990 & 0.22094 & 0.22282 & 0.8509 \\
\hline .995 & 0.25436 & 0.25655 & 0.8609 \\
\hline .9975 & 0.28829 & 0.28896 & 0.2324 \\
\hline .999 & 0.33374 & 0.33544 & 0.5093 \\
\hline \multicolumn{4}{|l|}{$\kappa=.7$} \\
\hline .50 & 0.04394 & 0.04370 & 0.5461 \\
\hline .75 & 0.06358 & 0.06316 & 0.6605 \\
\hline .80 & 0.06992 & 0.06943 & 0.7008 \\
\hline .85 & 0.07822 & 0.07757 & 0.8309 \\
\hline .90 & 0.09020 & 0.08937 & 0.9201 \\
\hline .95 & 0.11145 & 0.11009 & 1.2202 \\
\hline .975 & 0.13357 & 0.13176 & 1.3550 \\
\hline .990 & 0.16385 & 0.16184 & 1.2267 \\
\hline .995 & 0.18734 & 0.18491 & 1.2971 \\
\hline .9975 & 0.21120 & 0.20847 & 1.2926 \\
\hline .999 & 0.24317 & 0.24069 & 1.0198 \\
\hline \multicolumn{4}{|l|}{$\kappa=1$} \\
\hline .50 & 0.04115 & 0.04079 & 0.8748 \\
\hline .75 & 0.05724 & 0.05650 & 1.2928 \\
\hline .80 & 0.06215 & 0.06128 & 1.3998 \\
\hline .85 & 0.06840 & 0.06732 & 1.5789 \\
\hline .90 & 0.07710 & 0.07574 & 1.7639 \\
\hline .95 & 0.09185 & 0.08991 & 2.1121 \\
\hline .975 & 0.10653 & 0.10415 & 2.2341 \\
\hline .990 & 0.12590 & 0.12298 & 2.3193 \\
\hline .995 & 0.14055 & 0.13731 & 2.3052 \\
\hline .9975 & 0.15519 & 0.15121 & 2.5645 \\
\hline .999 & 0.17454 & 0.17110 & 1.9708 \\
\hline
\end{tabular}

Table 4 shows the asymptotic critical values and critical values computed by extrapolating the simulated critical values in Section 3 to $n=\infty$. It is shown that the relative differences are smaller than $3 \%$ for all critical values. Here we use three values for $\kappa$. This is true for all $\kappa \in[0.1,10.0]$, which covers most cases in practice.

One might wonder what happens for large and small $\kappa$. In fact, the asymptotic covariance function $\rho(s, t)$ becomes stable for larger or small $\kappa$. The following limits can be easily derived from (20) and (23) for both Case A and Case B:

$$
\begin{aligned}
& \rho(\kappa ; s, t)_{\kappa \rightarrow 0}=\min \{s, t\}-s t \\
& -(1-s)(1-t) \log (1-s) \log (1-t), \\
& \rho(\kappa ; s, t)_{\kappa \rightarrow \infty}=\min \{s, t\}-s t \\
& -s t \log (s) \log (t),
\end{aligned}
$$

$$
\begin{aligned}
& \rho_{1}(\kappa ; s, t)_{\kappa \rightarrow 0}=\min \{s, t\}-s t \\
& -(1-s)(1-t) \log (1-s) \log (1-t), \\
& \rho_{1}(\kappa ; s, t)_{\kappa \rightarrow \infty}=\min \{s, t\}-s t \\
& -s t \log (s) \log (t),
\end{aligned}
$$

which are finite functions of $s$ and $t$. This convergence means that as $\kappa \rightarrow 0$ or $\kappa \rightarrow \infty$, the EDF statistics have stable critical values.

\section{APPLICATIONS}

In this section, we apply the tests developed in the previous sections on some popularly studied financial factors on portfolio returns. Fama and French (1993 [5], 1995 [6], 1996 [7]) proposed factors based on zero-investment portfolios to explain asset portfolio pricing. The SMB factor represents the return from a zero-investment portfolio which is long on small stocks and short on large stocks. The HML factor represents the return from a zero-investment portfolio which is long on high book-to-market stocks and short on low book-to-market stocks. The UMD factor represents the return from a zero-investment portfolio which is long on high prior return stocks and short on low prior return stocks. The MKTEK factor represents a broad market portfolio return in excess of the risk-free (RF) return. Fama and French (1993 [5], 1995 [6], 1996 [7]) showed that these facotrs catch returns for most portfolios.

It has been noticed that these factors exhibit large variability over time. It is interesting to see what distribution these factors follow if ignoring the time dependence. We take the monthly data from January 2000 to December 2007. The selection of the data is somewhat ad hoc by only considering a middle sample size close to 100 . Figures 1, 2, 3, 4, 5 show histograms of the 4 factors and the risk-free return (RF) in the same period. Fitted three-parameter asymmetric Laplace distribution using the MLE method in Section 2 is super-imposed on each histogram. Test statistics and corresponding $p$-values are shown in Table 5. Except the riskfree return, all tests of an asymmetric Laplace distribution for the 4 factors accept the null hypothesis with high $p$-values.

These results partially confirm a popular observation that market return related variables have high frequency around some median values, sharp decreasing frequency around these median values, and higher frequency for extreme values than the normal distribution. Among the 4 factors accepting the asymmetric Laplace null hypotheses, MKTEX and UMD show asymmetry (skewed on the left), while the other two look more symmetric. These observations are consistent with the conclusion by Bakshi, Madan, and Panayotov $(2010,[2])$ that market crashes are more severe in intensity than rallies, and have higher arrival rates. The riskfree return rejects the null hypotheses with high significance. The histogram in Figure 4 shows a pattern of double peaks, which would violate the steep unique peak with the Laplace distribution. 


\section{Histogram of mktex}

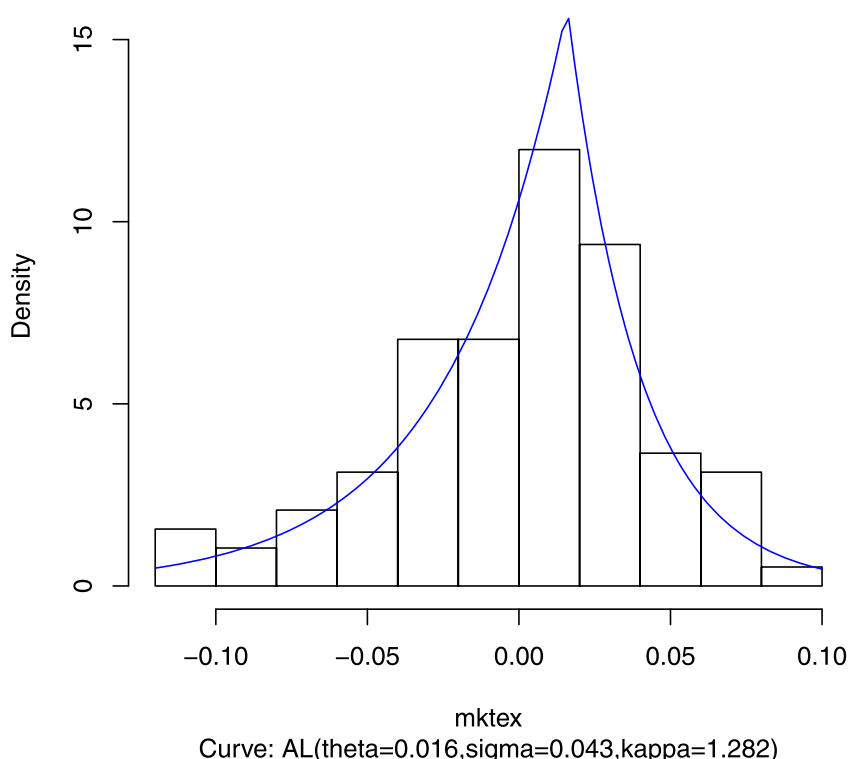

Figure 1. Three-parameter Asymmetric Laplace Fit of mktex.

Histogram of smb

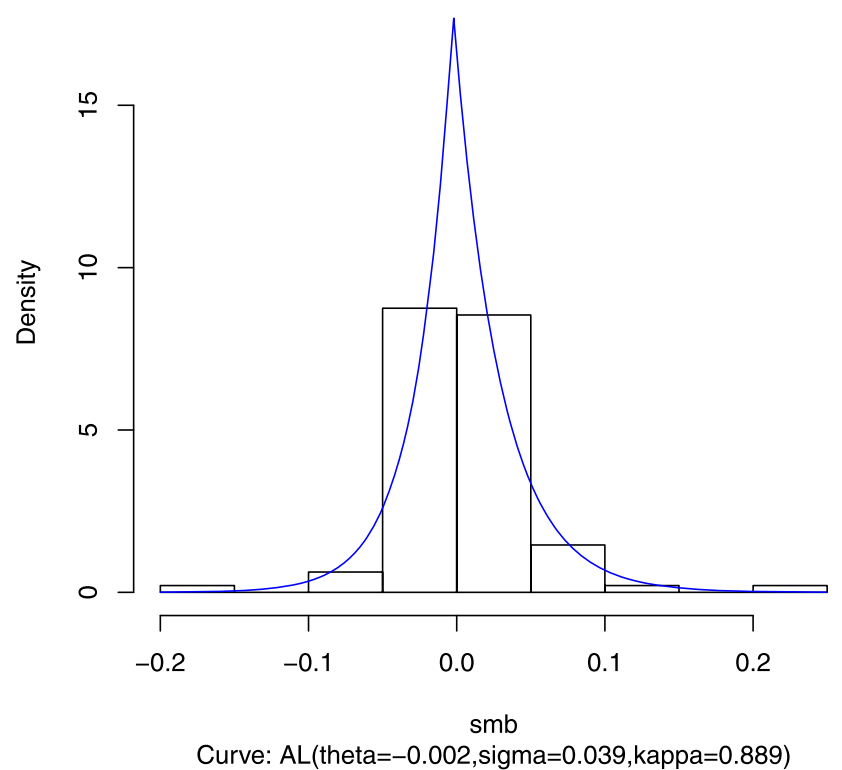

Figure 2. Three-parameter Asymmetric Laplace Fit of smb.

\section{POWER STUDY}

The power of the three EDF tests has been examined by Monte Carlo studies. Random samples of size $n$ were taken from a broad range of distributions; these include such distributions as the $T$ and gamma, which are used in the lit-
Histogram of hml

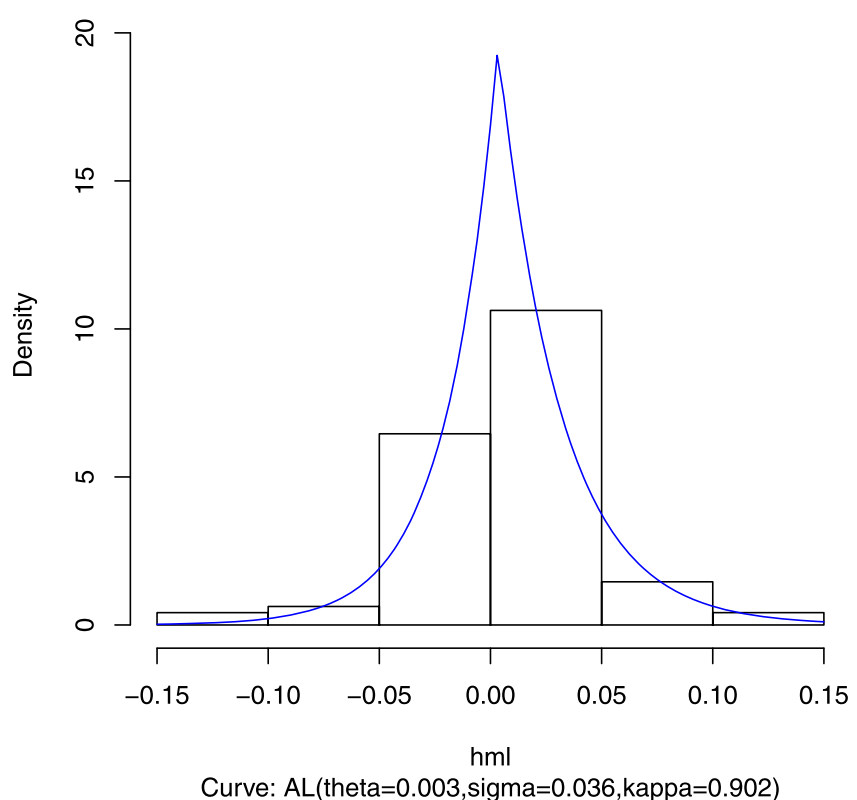

Figure 3. Three-parameter Asymmetric Laplace Fit of hml.

Histogram of $\mathbf{r f}$

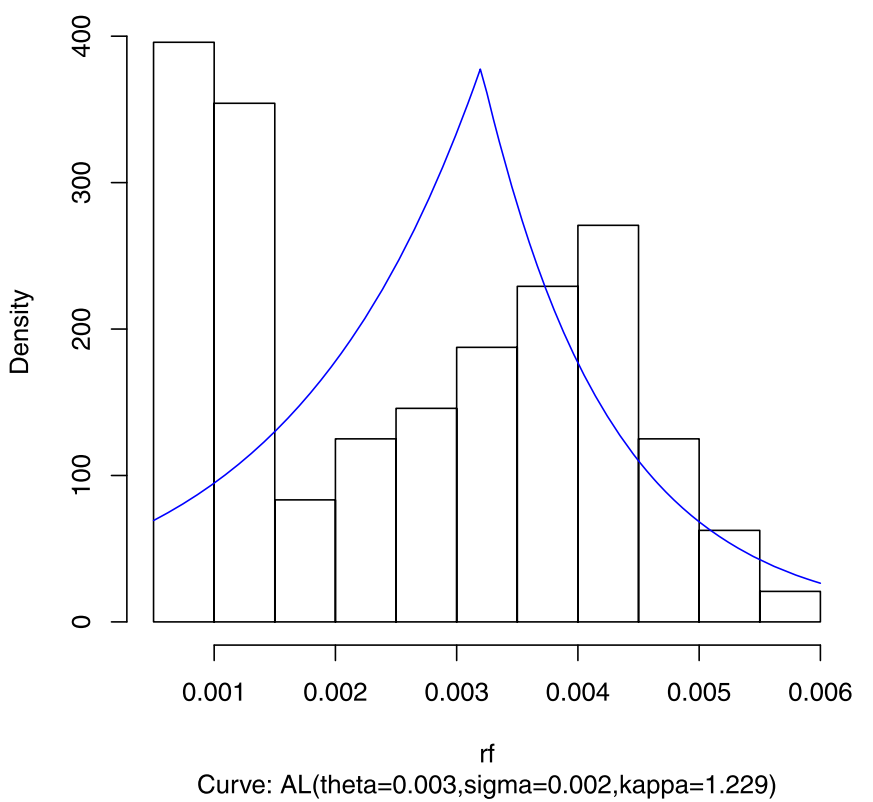

Figure 4. Three-parameter Asymmetric Laplace Fit of rf.

erature as practical alternatives to the Laplace distribution as a model. For the $T$ alternative, $T$ distributions with degrees of freedom of 2 and 4, $T(2)$ and $T(4)$ are used. For the gamma alternative, the gamma distribution with shape 5 and scale $1(G(5,1))$ is used. 


\section{Histogram of umd}

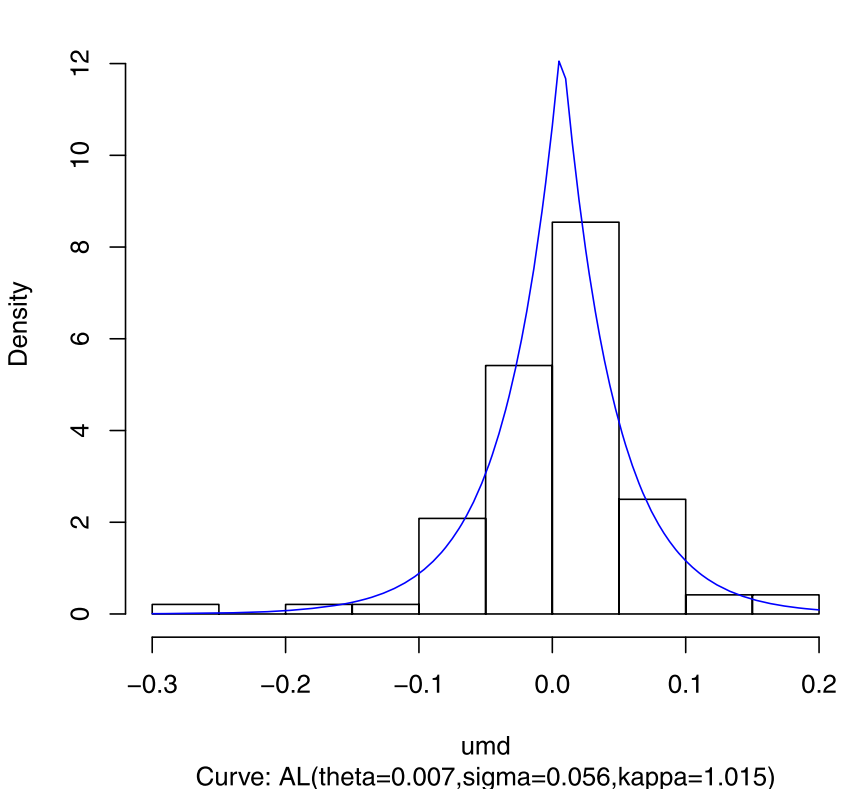

Figure 5. Three-parameter Asymmetric Laplace Fit of umd.

The lognormal distribution and two families of transformed distributions, $S_{u}$ and $S_{b}$, are used. These families belong to the Johnson system, which are distributions based on a transformed normal variate,

$$
Z=\xi+\delta T(X)
$$

where $Z \sim N(0,1), \xi$ and $\delta$ are location and scale, and $T$ is a transformation of the variate $X$. See Chapter 12 of Johnson, Kotz, and Balakrishnan (1994, [11]) for details. Five distributions were selected from these two families $S_{u}(\xi, \delta)$ and $S_{b}(\xi, \delta)$ with different combinations of $\xi$ and $\delta$ as alternatives in our power study. All these alternatives and some other commonly used alternatives are described in Table 6 .

These distributions were then tested against the threeparameter Laplace distribution using the three EDF statistics. Table 7 shows the rounded percentage of 10,000 samples declared significant when the test level is $\alpha=0.1$.

For alternatives with bounded support, $B e(2,3), U(0,1)$, and the two distributions in the $S_{b}$ family, the power of EDF tests depends on the shape of the alternatives. The two $S_{b}$ distributions are a little more similar in shape to the Laplace distribution. Table 7 shows that EDF tests have lower power with these two alternatives. For alternatives with unbounded support, the power of EDF tests depends heavily on the tail behavior of the alternatives. EDF tests have higher power for alternatives with lighter tails, such as the normal and lognormal distributions. The three $S_{u}$ distributions and the two $T$ distributions have heavier tails. Among them, $T(4)$ has the heaviest tail and also sharpest peak in the center, thus the lowest power. One interesting case is
Table 5. Goodness-of-Fit Tests for Asymmetric Laplace Distribution

\begin{tabular}{|c|c|c|c|c|}
\hline$\overline{\text { Test }}$ & \multicolumn{2}{|c|}{ Statistic } & \multicolumn{2}{|c|}{$p$-Value } \\
\hline \multicolumn{5}{|c|}{ mktex } \\
\hline Kolmogorov-Smirnov & $\mathrm{D}$ & 0.6965 & $\operatorname{Pr}>\mathrm{D}$ & 0.197 \\
\hline Cramér-von Mises & W-Sq & 0.0644 & $\operatorname{Pr}>\mathrm{W}-\mathrm{Sq}$ & 0.203 \\
\hline Anderson-Darling & $\mathrm{A}-\mathrm{Sq}$ & 0.3903 & $\mathrm{Pr}>\mathrm{A}-\mathrm{Sq}$ & 0.245 \\
\hline \multicolumn{5}{|c|}{$\mathrm{smb}$} \\
\hline Kolmogorov-Smirnov & $\mathrm{D}$ & 0.3446 & $\operatorname{Pr}>D$ & $>0.5$ \\
\hline Cramér-von Mises & W-Sq & 0.0195 & $\mathrm{Pr}>\mathrm{W}-\mathrm{Sq}$ & $>0.5$ \\
\hline Anderson-Darling & $\mathrm{A}-\mathrm{Sq}$ & 0.2342 & $\operatorname{Pr}>\mathrm{A}-\mathrm{Sq}$ & $>0.5$ \\
\hline \multicolumn{5}{|c|}{$\mathrm{hml}$} \\
\hline Kolmogorov-Smirnov & $\mathrm{D}$ & 0.5725 & $\operatorname{Pr}>\mathrm{D}$ & 0.475 \\
\hline Cramér-von Mises & $\mathrm{W}-\mathrm{Sq}$ & 0.0275 & $\mathrm{Pr}>\mathrm{W}-\mathrm{Sq}$ & $>0.5$ \\
\hline Anderson-Darling & $\mathrm{A}-\mathrm{Sq}$ & 0.2419 & $\operatorname{Pr}>\mathrm{A}-\mathrm{Sq}$ & $>0.5$ \\
\hline
\end{tabular}

$\mathrm{rf}$

Kolmogorov-Smirnov

Cramér-von Mises

$\mathrm{D}$

$\operatorname{Pr}>\mathrm{D} \quad<0.001$

W-Sq $\quad 0.5915$

$\mathrm{A}-\mathrm{Sq} \quad 3.7324$

$\mathrm{Pr}>\mathrm{W}-\mathrm{Sq} \quad<0.001$

$\mathrm{Pr}>\mathrm{A}-\mathrm{Sq} \quad<0.001$

umd

\begin{tabular}{lllll} 
Kolmogorov-Smirnov & D & 0.6258 & $\mathrm{Pr}>\mathrm{D}$ & 0.325 \\
Cramér-von Mises & W-Sq & 0.0583 & $\mathrm{Pr}>\mathrm{W}-\mathrm{Sq}$ & 0.225 \\
Anderson-Darling & A-Sq & 0.3470 & $\mathrm{Pr}>\mathrm{A}-\mathrm{Sq}$ & 0.344 \\
\hline
\end{tabular}

Table 6. Alternative Distributions

\begin{tabular}{ll}
\hline \hline$N(0,1)$ & the standard normal \\
$L N(0,1)$ & lognormal with location 0 and scale 1 \\
$T(2)$ & $t$ with 2 degrees \\
$T(4)$ & $t$ with 4 degrees \\
$G(5,1)$ & gamma with shape 5 and scale 1 \\
$B e(2,3)$ & beta with shape 2 and shape 3 \\
$U(0,1)$ & standard uniform \\
$S_{u}(0,2)$ & $S_{u}$ with $\xi=0$ and $\delta=2$ \\
$S_{u}(1,2)$ & $S_{u}$ with $\xi=1$ and $\delta=2$ \\
$S_{u}(1,1)$ & $S_{u}$ with $\xi=1$ and $\delta=1$ \\
$S_{b}(0,2)$ & $S_{b}$ with $\xi=0$ and $\delta=2$ \\
$S_{b}(1,2)$ & $S_{b}$ with $\xi=1$ and $\delta=2$ \\
\hline
\end{tabular}

the gamma alternative $G(5,1)$. It has a similar tail as the Laplace distribution; however, its peak is much less sharper than the Laplace distribution, thus it yields a higher power.

One interesting observation with the three $S_{u}$ distributions is that Table 7 shows that the Anderson-Darling statistic $A^{2}$ has lower power, especially with $S_{u}(1,1)$ and smaller samples, while it dominates the other two for all other alternatives. 
Table 7. Power Comparison

\begin{tabular}{|c|c|c|c|c|c|c|c|c|}
\hline & Sam & $\overline{\mathrm{eSi}}$ & & & & & & \\
\hline & $\mathrm{n}$ & 35 & 50 & 75 & 100 & 125 & 150 & 200 \\
\hline$N(0,1)$ & & & & & & & & \\
\hline & $D$ & 24 & 33 & 48 & 61 & 71 & 79 & 89 \\
\hline & $W^{2}$ & 26 & 39 & 59 & 75 & 85 & 91 & 97 \\
\hline & $A^{2}$ & 25 & 39 & 61 & 76 & 86 & 92 & 98 \\
\hline$L N(0, .1)$ & & & & & & & & \\
\hline & $D$ & 20 & 32 & 44 & 57 & 67 & 76 & 87 \\
\hline & $W^{2}$ & 23 & 38 & 56 & 71 & 81 & 89 & 96 \\
\hline & $A^{2}$ & 24 & 40 & 58 & 74 & 84 & 91 & 97 \\
\hline$T(2)$ & & & & & & & & \\
\hline & $D$ & 31 & 36 & 44 & 49 & 54 & 59 & 66 \\
\hline & $W^{2}$ & 32 & 40 & 47 & 54 & 60 & 66 & 74 \\
\hline & $A^{2}$ & 34 & 43 & 52 & 60 & 66 & 72 & 80 \\
\hline$T(4)$ & & & & & & & & \\
\hline & $D$ & 14 & 16 & 19 & 22 & 25 & 29 & 35 \\
\hline & $W^{2}$ & 13 & 17 & 21 & 25 & 29 & 34 & 42 \\
\hline & $A^{2}$ & 13 & 16 & 21 & 25 & 30 & 34 & 42 \\
\hline$G(5,1)$ & & & & & & & & \\
\hline & $D$ & 20 & 25 & 34 & 44 & 54 & 64 & 77 \\
\hline & $W^{2}$ & 22 & 29 & 41 & 55 & 66 & 77 & 89 \\
\hline & $A^{2}$ & 25 & 34 & 49 & 63 & 74 & 84 & 93 \\
\hline$B e(2,3)$ & & & & & & & & \\
\hline & $D$ & 35 & 48 & 68 & 82 & 91 & 95 & 99 \\
\hline & $W^{2}$ & 43 & 62 & 84 & 95 & 98 & 99 & 99 \\
\hline & $A^{2}$ & 48 & 70 & 91 & 98 & 99 & 99 & 99 \\
\hline$U(0,1)$ & & & & & & & & \\
\hline & $D$ & 67 & 78 & 95 & 99 & 99 & 100 & 100 \\
\hline & $W^{2}$ & 85 & 94 & 99 & 99 & 100 & 100 & 100 \\
\hline & $A^{2}$ & 91 & 96 & 100 & 100 & 100 & 100 & 100 \\
\hline$S_{u}(0,2)$ & & & & & & & & \\
\hline & $D$ & 17 & 21 & 28 & 37 & 44 & 50 & 62 \\
\hline & $W^{2}$ & 17 & 22 & 32 & 43 & 53 & 61 & 74 \\
\hline & $A^{2}$ & 16 & 20 & 30 & 41 & 51 & 59 & 73 \\
\hline$S_{u}(1,2)$ & & & & & & & & \\
\hline & $D$ & 20 & 25 & 34 & 42 & 49 & 56 & 67 \\
\hline & $W^{2}$ & 19 & 27 & 37 & 48 & 56 & 64 & 76 \\
\hline & $A^{2}$ & 17 & 23 & 33 & 44 & 52 & 61 & 73 \\
\hline$S_{u}(1,1)$ & & & & & & & & \\
\hline & $D$ & 42 & 47 & 53 & 58 & 63 & 68 & 75 \\
\hline & $W^{2}$ & 42 & 47 & 53 & 60 & 65 & 70 & 77 \\
\hline & $A^{2}$ & 37 & 39 & 44 & 52 & 57 & 63 & 71 \\
\hline$S_{b}(0,2)$ & & & & & & & & \\
\hline & $D$ & 29 & 41 & 58 & 73 & 82 & 89 & 96 \\
\hline & $W^{2}$ & 32 & 50 & 73 & 87 & 93 & 97 & 99 \\
\hline & $A^{2}$ & 33 & 53 & 76 & 90 & 95 & 98 & 99 \\
\hline$S_{b}(1,2)$ & & & & & & & & \\
\hline & $D$ & 28 & 37 & 53 & 67 & 79 & 85 & 94 \\
\hline & $W^{2}$ & 31 & 45 & 67 & 82 & 91 & 96 & 99 \\
\hline & $A^{2}$ & 32 & 49 & 82 & 87 & 94 & 97 & 99 \\
\hline
\end{tabular}

\section{CONCLUSION AND GUIDELINES}

Goodness-of-fit tests for the three-parameter Laplace distribution have been developed based on the EDF statistics $\sqrt{n} D, W^{2}$, and $A^{2}$. Asymptotic critical values for these test statistics are derived. For finite samples, these statistics are computed with estimated parameters based on MLE. The MLE is reliable and efficient. It can be used to simulate the critical values for these statistics in finite sample cases. These simulated critical values match asymptotic critical values computed for the two quadratic EDF statistics $W^{2}$ and $A^{2}$ with sufficient accuracy. An application of these tests has been demonstrated and their power has been studied.

To efficiently summarize and use these simulated critical values for finite samples, simple polynomial approximations have been developed. This approximation is better than or as good as the traditional linear interpolation, although an estimated shape parameter must be used in those polynomials. One conservative suggestion is to use an estimated confidence interval for the shape parameter instead of a single value. By using the two end points of this interval, one can get intervals of the critical values with these polynomials. $p$-values can be computed according to whether EDF statistics fall into these intervals.

Since the MLE of the three-parameter Laplace distribution is not regular, here are some guidelines on how to use these proposed EDF tests in practice:

1. The sample size should not be less than 35. Although the MLE procedure may still find a solution for data with less than 35 observations, the power of the EDF tests may be too low.

2. The estimated shape parameter should be within the range $[0.1,10.0]$. This range should cover all the cases in practice.

3. To be conservative, use an estimated interval of the shape parameter to compute $p$-values.

\section{ACKNOWLEDGMENT}

The author would like to thank editors and referees for their helpful comments.

Received 14 July 2013

\section{REFERENCES}

[1] Ayebo, A. and Kozubowski, T. J. (2003). An asymmetric generalization of Gaussian and Laplace laws, Journal of Probability and Statistical Science, 1, 187-210.

[2] Bakshi, G., Madan, D., and Panayotov, G. (2010). Deducing the implications of jump models for the structure of stock market crashes, rallies, jump arrival rates, and extremes, JBES, 28, 380396. MR2723607

[3] Chen, C. (2002). Tests for the goodness-of-fit of the Laplace distribution, Communication in Statistics - Simulation and Computation, 31, 159-174. MR1894272

[4] Durbin, J. (1973). Weak convergence of the sample distribution function when parameters are estimated, Ann. Statist., 1, 279290. MR0359131

[5] Fama, E. F. and French, K. R. (1993). Common risk factors in the returns on stocks and bonds, Journal of Financial Economics, 33, 3-56.

[6] Fama, E. F. and French, K. R. (1995). Size and book-to-market factors in earnings and returns, Journal of Finance, 50, 131-156.

[7] Fama, E. F. and French, K. R. (1996). Multifactor explanations of asset pricing anomalies, Journal of Finance, 51, 55-84. 
[8] Hartley, M. J. and Revankar, N. S. (1974). On the estimation of the Pareto law from underreported data, Journal of Econometrics, 2, 327-341. MR0652564

[9] Hinkley D. V. and Revankar, N. S. (1977). Estimation of the Pareto law from underreported data, Journal of Econometrics, 5, 1-11. MR0652697

[10] Imhof, J. P. (1961). Computing the distribution of quadratic forms in normal variables, Biometrika, 48, 419-426. MR0137199

[11] Johnson, N. L., Kotz, S., and Balakrishnan N. (1994). Continuous Univariate Distributions, Vol. 1, Wiley, New York. MR1299979

[12] Kotz, S., Kozubowski, T. J., and Podgórski, K. (2001). The Laplace Distribution and Generalizations, Birkhäuser, Boston. MR1935481

[13] Kozubowski, T. J. and Podgórski, K. (1999). A class of asymmetric distribution, Actuarial Research Clearing House, 1, 113134.

[14] Kozubowski, T. J. and Podgórski, K. (2001). Asymmetric Laplace laws and modeling financial data, Math. Comput. Modelling, 34, 1003-1021. MR1858834
[15] LongstafF, F. A. (1994). Stochastic volatility and option valuation: a pricing-density approach, Preprint, The Anderson Graduate School of Management. UCLA.

[16] Madan, D. B., Carr, P., and Chang E. C. (1998). The Variance Gamma Process and Option Pricing, European Finance Review, 2, 74-105.

[17] Puig, P. and Stephens, M. A. (2000). Tests of fit for the Laplace distribution, with applications, Technometrics, 42, 417424. MR1802472

[18] Stephens, M. A. (1986). Tests based on EDF statistics, in Goodness-of-fit Techniques (eds, R. B. D'Agostino and M. A. Stephens), ch. 4. Marcel Dekker, New York. MR0874534

\section{Colin Chen}

Bank of America and Georgetown University

Boyds, MD 20841

USA

E-mail address: chennorthc@gmail.com 